\title{
Tools for Community-Oriented Primary Care: A Process for Linking Practice and Community Data
}

\author{
Thomas M. Mettee, MD, Kevin B. Martin, MD, and Robert L. Williams, MD, MPH
}

Background: Community-oriented primary care (COPC) is an increasingly attractive paradigm for primary care delivery. Further work is needed, however, to implement COPC in busy practice settings. This study reports a feasible method for linking practice and community health data for use in COPC.

Methods: Using one practice and its community as an example of the process, we sought data related to five common cancers. Data from readily accessible community sources were combined with practice morbidity data using commonly available computer hardware and software.

Results: We developed a user-friendly database and maps showing rates and distribution of the example diseases. We also developed strategies to obtain complete case identification and to address confidentiality and proprietary concerns.

Conclusions: Understanding patterns of disease expression in the practice and the community is critical to the COPC process. Rapid, inexpensive methods for displaying these patterns, such as the database and maps described, must be accessible to clinicians if COPC is to move from theory into practice. Partnerships between health care providers and institutions can also help get this capability into the hands of clinicians. (J Am Board Fam Pract 1998;11:28-33.)

Rapidly developing trends in health care delivery have created an imperative for testing new approaches to care, and declining resources have emphasized the need for targeted and effective interventions, cost-effective care, and preventive care. Community-oriented primary care (COPC) is an approach that meets these criteria. COPC is designed to target community-specific needs, to make efficient use of resources, and to link preventive care in the practice with community-based prevention efforts. Indeed, some authors have described a COPC-like system as being the final stage of evolution of managed care environments. ${ }^{1,2}$

Despite the theoretic attractiveness of COPC, feasible methods for applying COPC to everyday practice have not yet been well described. Published examples of COPC have generally reported

Submitted, revised, 16 April 1997.

From the Chesterland Family Practice, Chesterland, Ohio (TMM), the Department of Family Medicine, Case Western Reserve University, Cleveland (TMM, KBM, RLW), the MetroHealth Center for Community Health, Cleveland (RLW), and the Department of Paediatrics, University of Natal, Durban, South Africa (RLW). Address reprint requests to Robert L. Williams, MD, MPH, Department of Family Medicine, 11001 Cedar, Suite 306, Cleveland, $\mathrm{OH} 44106$.

This study was supported by a Robert Wood Johnson Foundation generalist physician faculty scholarship (RLW), and by a Fulbright Senior Scholar Award (RLW). methods that are not realistic in most practice settings. A recent survey of family physicians emphasized that a principal reason for the limited application of COPC to date has been a lack of practical methods to implement it, ${ }^{3}$ and without practical methods of implementation, the efficacy of the COPC model cannot be validly tested.

COPC applies public health concepts to the delivery of primary care ${ }^{4,5}$ (Figure 1). An early step in this process is to review the community's health-related data. Recently, commonly available software has been applied for use in small-area mapping of health indicators, making it much more possible for practicing physicians to carry out this step. ${ }^{6,7}$ Objective health indicators are combined in a personal computer database. Using a geographic-mapping software program, their distribution can be displayed on a community map for easy review by the practitioner.

Another step in the COPC process is linking health data from the community to data from the practice. Comparing data from these two sources can help uncover health needs or unique patterns of disease in either the practice or the community. Practice data can also be compared with regional or national data. Each data source can be used as a window affording a different view on the community. Through these windows, a COPC practice might be able to (1) answer a clinical question, (2) 
assess service delivery for a specific condition, or (3) target an intervention.

We wanted to develop further methods for implementing COPC by designing a practical approach for carrying out this next step in the COPC process. The specific aims were to describe a feasible method of linking community and practice data using mapping capabilities; to analyze the process with attention to costs, benefits, and problems encountered; and to offer an example of its application.

To study the process, we chose to link morbidity data from a family practice to analogous data for the entire county (defined as the community) in which the practice was located and to 1990 census data. In our example the practice and community had a clinical question. The practice perceived an increase in cases of common types of cancer. The community had also voiced widespread concern about a possible increasing incidence in cancer. This concern was coupled with fears among some community residents that such an increase could be linked to industrial contamination of county groundwater supplies.

\section{Methods}

The study goal was to examine tools that would provide physicians with information about both the practice and the surrounding community while requiring a minimal commitment of time and material resources. For this project we used an inexpensive menu-driven database package (Alpha $4^{8}$ ) and a desktop mapping package (MapInfo ${ }^{9}$ ). The community health database was developed on a low-end IBM-compatible personal notebook computer.

\section{Study Setting}

The county studied was Geauga County, a suburban-rural county in northeastern Ohio with a population in 1990 of 81,129 . The practice, located in the northwestern portion of the county, had an estimated 5500 patient visits per year.

\section{Linkage of Health Indicators}

Based on the community and practice concerns about an increase in cases of cancer, distributions of five common cancers from 1990 to 1992 were used as a model for developing the COPC method. Data for cancer cases in the county were obtained in electronic format from the cancer reg-

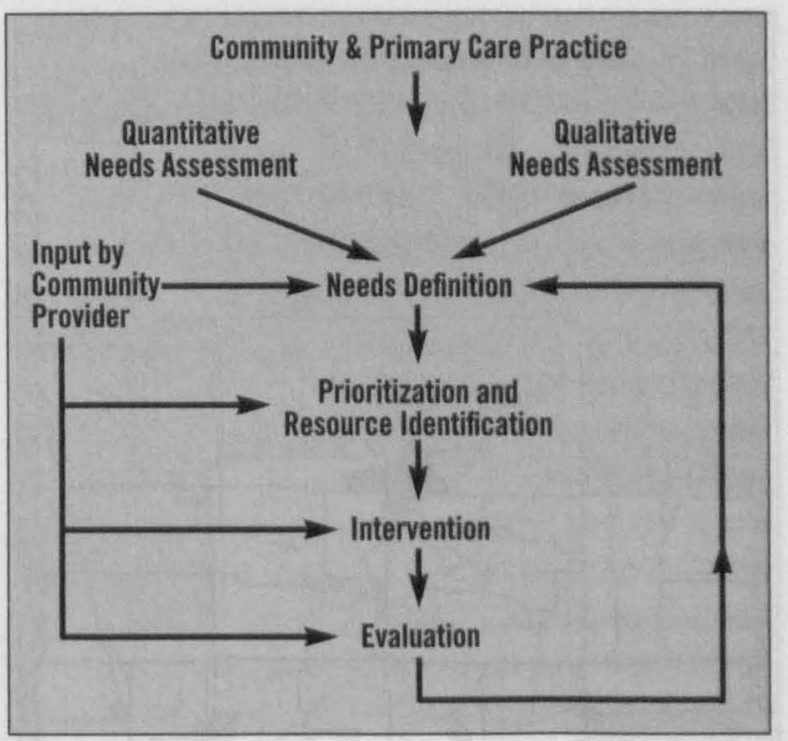

Figure 1. Schematic diagram of the COPC process.

istry maintained at the county hospital and from the regional cancer registry. Personal data were not collected. To calculate population rates, block level county census data were obtained in electronic format from a local branch of the public library.

Information on cancer cases in the practice during the same period was collected by an electronic search of billing records. The list obtained was supplemented by manual review of referral letters requesting diagnostic evaluation for patients subsequently found to have cancer. Deceased patients' charts were also reviewed for a cancer diagnosis. Finally, the hospital cancer registry was reviewed for practice patients.

All data were electronically imported into the database and linked to the mapping software using address identifiers. Maps were then produced using selected variables.

\section{Results}

The final product was a database and associated maps related to the target diagnoses. Examples of the types of maps that can be produced are shown in Figures 2 through 5. Figure 2 is a map of the density of the at-risk population in the county. In Figure 3 the individual cases of breast cancer in the county are plotted against the density of the at-risk population. These data were then used to develop Figure 4 to show the geographic distribution of the incidence of cancer among the at-risk population. Finally, the practice cases were plotted as shown on Figure 5. 


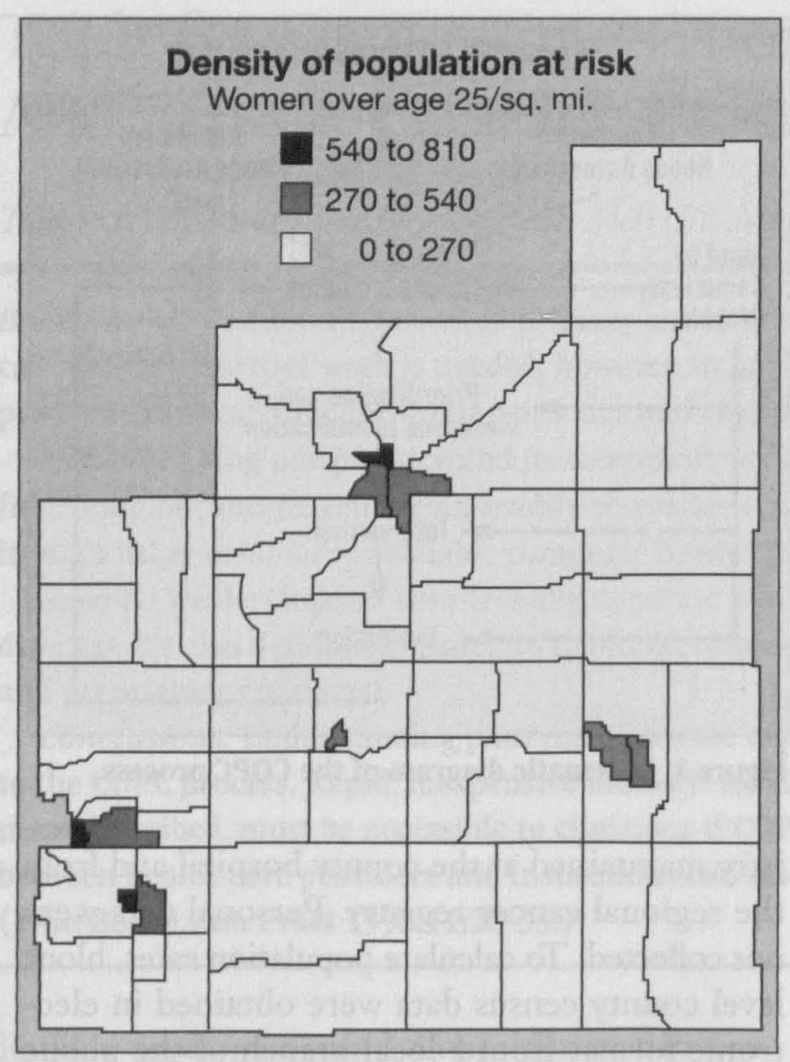

Figure 2. Example map produced from COPC database showing Geauga County with distribution per square mile of women older than 25 years, the population at risk for breast cancer. Source: 1990 county census. Mapped to census block groups.

Maps such as these are clinically useful because they allow the COPC practitioner to compare practice and community incidences and prevalences of selected diseases, geographic distributions of cases, and possible high-risk segments of the practice or community. In the specific example described, the maps formed the basis of discussions at both the local hospital and in the community about the distribution of cancer in the practice and within the community, which ultimately led to reassurance about the perceived problem.

\section{Resource Requirements}

This project used hardware with capabilities similar to or less than what is found in many practices today. Currently available database software (ranging from \$100 to \$150) can rapidly combine and handle both large data sets and individual records within each set. Mapping packages $(\$ 500$ to $\$ 1800)$ make it possible to merge information from various sources, either through direct import or by using compatible database software, and dis-

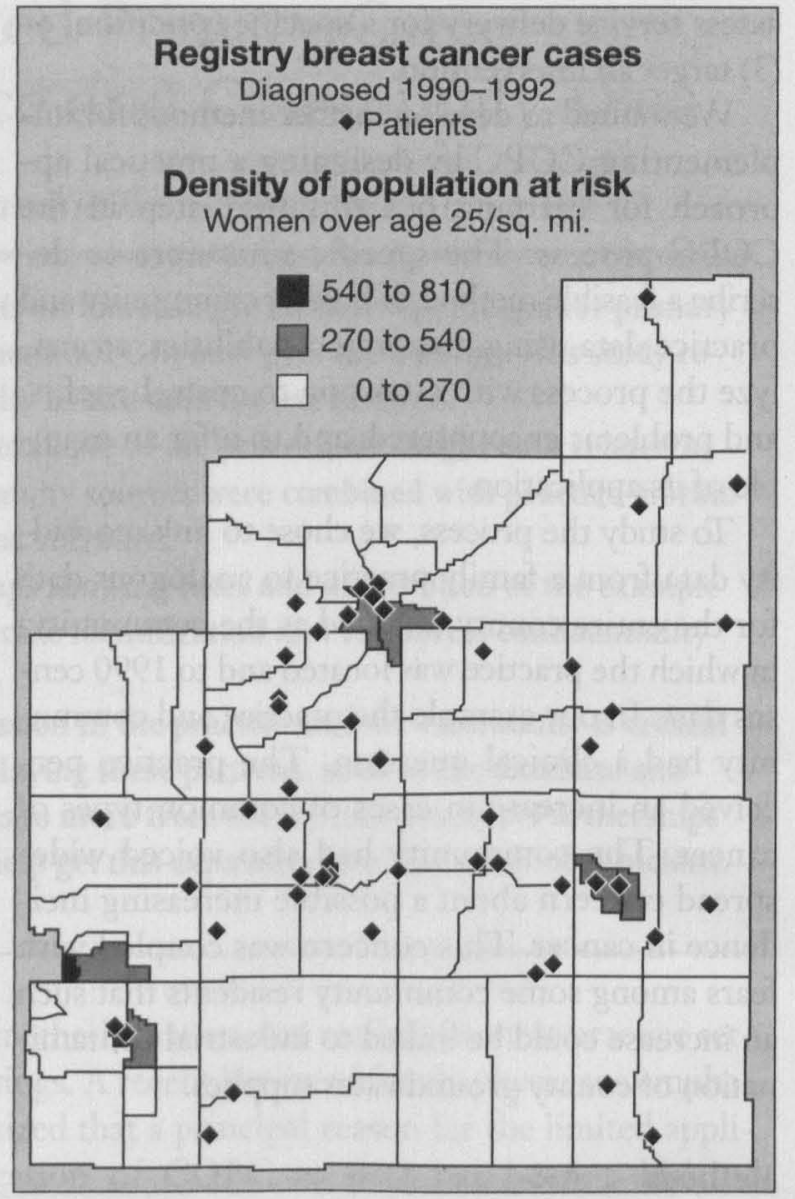

Figure 3. Example map produced from COPC database showing Geauga County with 3 years of new cases of breast cancer displayed against distribution of women older than $25 \mathrm{y} / \mathrm{sq}$ mi. Source: 1990 county census and regional cancer registry for cancer cases diagnosed from 1990-1992.

play it on a map of the community. Some packages are also capable of mapping individual cases as address points. Geographic software capabilities are developing rapidly, and prices are declining.

Personnel costs involved both planning and implementation time, each of which took approximately 20 to 30 hours. An additional 10 to 20 hours were spent in using the software and organizing the data to produce the desired reports or maps. This time could be substantially reduced if the capability were an integral part of practice operations.

\section{Obstacles Encountered}

The specific problems encountered in this project are illustrative of the general types of obstacles that can be expected elsewhere. A principal problem was obtaining complete case information at 


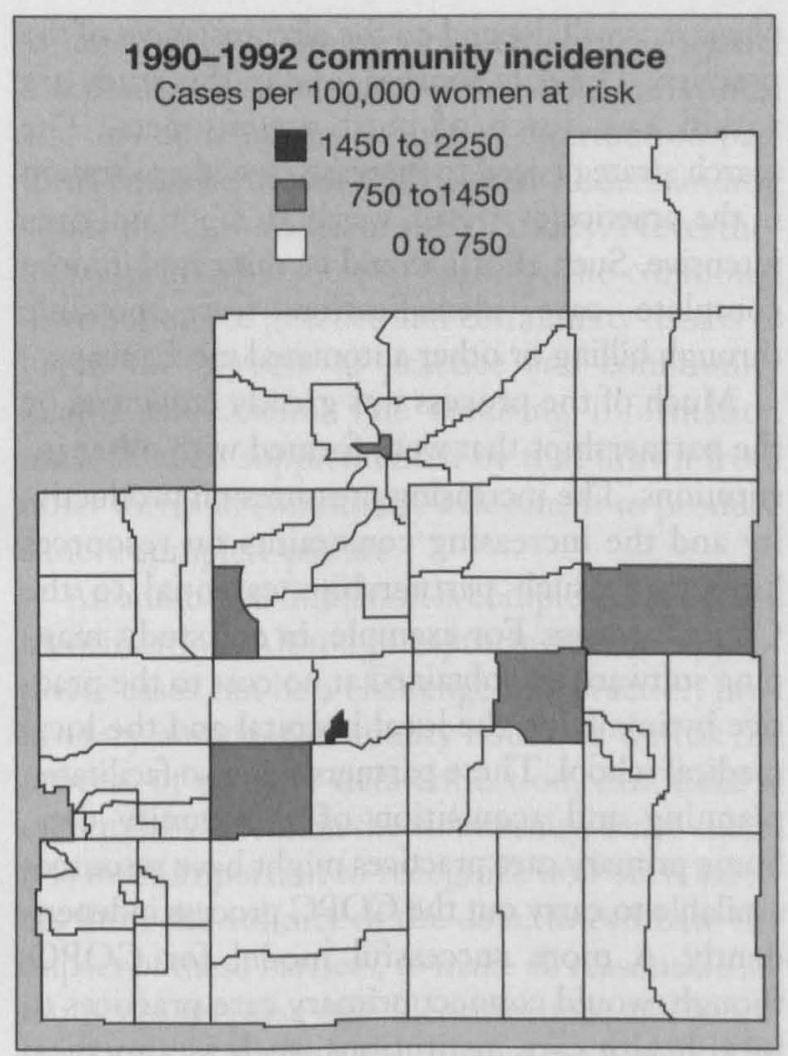

Figure 4. Example map produced from COPC database showing Geauga County with 3-year incidence of breast cancer among the county at-risk population. Source: 1990 county census and regional cancer registry (1990-1992). Mapped to census block groups.

both community and practice levels. No data source contained complete data sets. Efforts to increase case identification led to two further problems in data collection: confidentiality and ownership. Obtaining address-level data for mapping purposes required written consent from each hospital for data release because of worries about breach of patient confidentiality. Furthermore, proprietary issues (ownership of cancer patient data) had to be negotiated to obtain data from one facility. Such proprietary and confidentiality concerns are unlikely to be unique to the area studied.

Using the practice billing database, we found only about 75 percent of target cases of cancer. This failure to capture all cases exemplifies a problem with the clinical application of data collected for billing purposes. If the condition of interest is not a billing diagnosis, case identification could suffer. Although the practice billed for care using other codes, billing for costs of cancer management often occurred through other specialists.

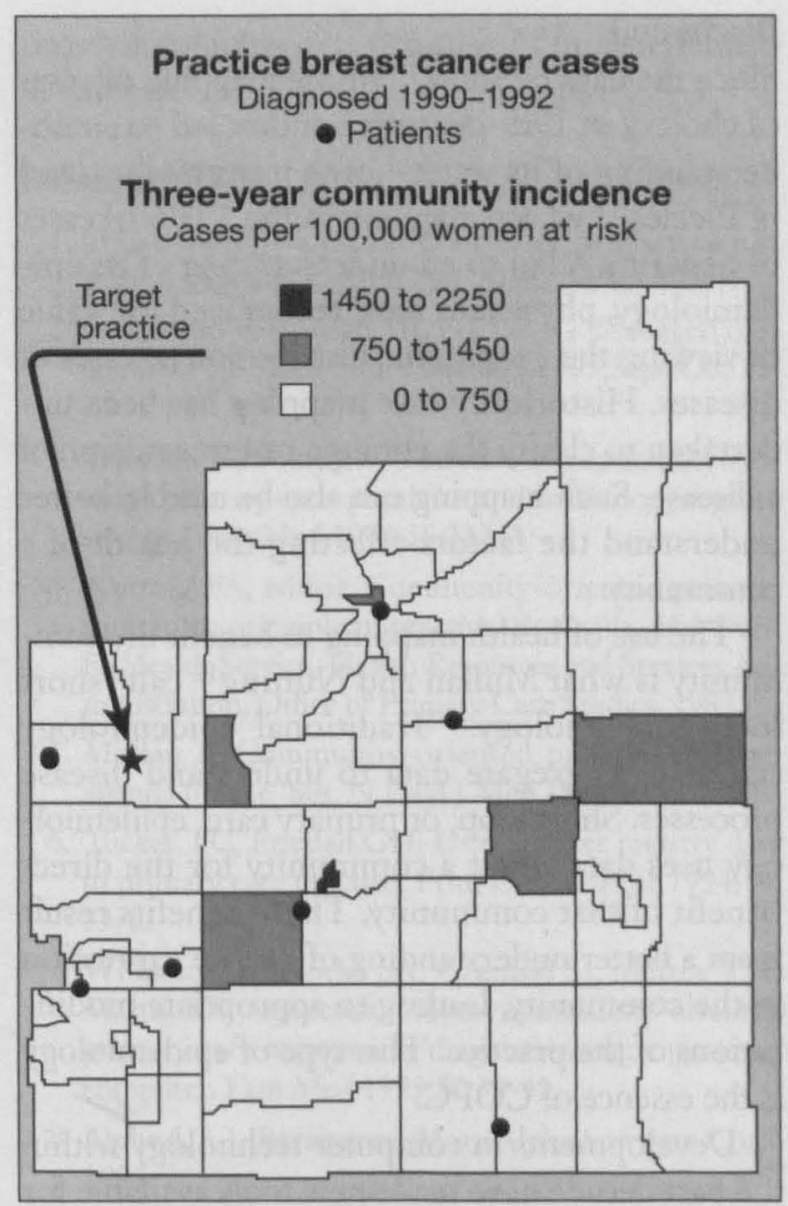

Figure 5. Example map produced from COPC database showing Geauga County with 3-year incident cases of breast cancer in the practice displayed against 3-year incidence of breast cancer among the county at-risk population. Source: 1990 county census, regional cancer registry (1990-1992), and practice billing records (1990-1992).

\section{Strategies to Overcome Obstacles}

Access to data can be improved by forming alliances with a range of institutions in the community. By sharing results with each institution and including their data needs during project planning, it was possible to forge partnerships that transcended proprietary issues. Confidentiality concerns were resolved through discussing the use of the data and agreeing on the format for the maps and databases.

Any problems encountered when searching for target cases in the practice database would be site specific but are probably not unique. A number of business software packages designed for clinical practices, however, now make it possible to enter demographic descriptors and nonbilling encounters. 


\section{Discussion}

Since the days of Snow, ${ }^{10}$ whose mapping of cases of cholera in 19th-century London led to an understanding of its water-borne transmission, and of Pickles, ${ }^{11}$ whose mapping in the 1930 s of cases of hepatitis A led to an understanding of its epidemiology, physicians have recognized the value of viewing the geographic distribution of cases of diseases. Historically case mapping has been undertaken to clarify the etiology or transmission of a disease. Such mapping can also be used to better understand the factors affecting the health of a community.

The use of health mapping to benefit the community is what Mullan and Nutting ${ }^{12}$ call "short loop epidemiology." Traditional epidemiology strives to aggregate data to understand disease processes. Short loop, or primary care, epidemiology uses data about a community for the direct benefit of that community. These benefits result from a better understanding of disease expression in the community, leading to appropriate modifications of the practice. This type of epidemiology is the essence of COPC.

Developments in computer technology within the past decade have made new tools available for COPC. We have described a process using commonly available and relatively inexpensive hardware and software to link and geographically display community and practice data. In the study example, practice and community data were linked to answer clinical questions about the incidence and distribution of specific cases of cancer. Any disease or condition about which community-based data are available could be studied in the same way.

The same process could also be followed to support specific interventions. For instance, by determining which portions of the practice or community populations get late prenatal care or are deficient in childhood immunizations, the practice or community could select specific groups of patients for intensive education. Depending on the condition studied, maps can be used to establish distribution patterns for incidence, prevalence, and change with time; to single out high-risk segments of the population; and to display possible etiologic factors in practice and community populations.

Although mapping health data is clearly valuable, whether this process is feasible for practicing physicians will depend on the circumstances of the practice. The data sources used in this study are within easy reach of most practitioners. The search strategy used to increase case identification in the practice, however, was both labor and time intensive. Such efforts would be mitigated if more complete case identification were possible through billing or other automated mechanisms.

Much of the process was greatly enhanced by the partnerships that were formed with other institutions. The increasing pressures on productivity and the increasing constraints on resources have made such partnerships essential to the COPC process. For example, in our study, mapping software was obtained at no cost to the practice by involving the local hospital and the local medical school. These partnerships also facilitated planning and acquisition of community data. Some primary care practices might have resources available to carry out the COPC process independently. A more successful model for COPC, though, would connect primary care practices to local health care institutions, such as a medical school or health departments. The time required to set up a database, such as the one described, could be eliminated with a centralized information-processing system.

As managed care organizations increasingly dominate primary care delivery, they are likely to become key partners in the COPC process. Capitated systems require the most efficient use of resources, which in turn requires that primary care match the health needs of the community and that delivery of preventive care be optimized. These goals will lead managed care organizations toward the COPC model and the development of databases such as the one described. Even so, the interests of managed care organizations and the data they generate will be almost exclusively centered on their own enrollees. Because most primary care physicians participate in many managed care plans, however, they (with assistance from other local health institutions) can still produce composite pictures of the practice and community populations. Furthermore, as managed care organizations attempt to pass financial risk on to practitioners, it will be in the interest of the practices to have these databases.

It is important to recognize the limitations of the data and the resulting maps. Generally there will be relatively few cases of most diseases in a 
practice or in a community. Temporal or spatial distributions can result from random variations, and any conclusions about any distribution patterns must be drawn with a clear understanding about the limitations of their validity. Nevertheless, a primary reason to examine the combined distributions of practice and community data is to begin the process of practice and community health assessment. The resulting information must then be supplemented by that drawn from other forms of community assessment to produce a more complete picture.

An additional limitation is completeness of case identification. Although capturing all the appropriate cases can be a challenge in a practice, proprietary and confidentiality issues, as well as the process of primary data collection, can make it even more difficult in the community. Once again, it is most important to recognize how such barriers limit the validity of the data, to estimate the impact of these barriers, to make all reasonable attempts to increase case identification, and to use great care in drawing any conclusions based solely on these data.

Despite these concerns, however, this information can be useful as one part of a community assessment for COPC. More extensive evaluation must await further research on the COPC models as a whole.

Findings from our study have shown that we now have the tools to compare and map practice and community disease patterns to support COPC. With the continuing development of practical methods for COPC, it might soon be possible to deliver its promise of more effective health care.

\section{References}

1. O'Connor PJ. Community-oriented primary care in a brave new world. Arch Fam Med. 1994;3:493-4.

2. Goldsmith JC, Goran MJ, Nackel JG. Managed care comes of age. Healthcare Forum J 1995;Sept/Oct:1523.

3. Williams R, Foldy SL. The state of community-oriented primary care: physician and residency program surveys. Fam Med 1994;26:232-7.

4. Nutting PA, editor. Community-oriented primary care: from principle to practice. Rockville, Md: Public Health Service, Health Resources and Services Administration, Office of Primary Care Studies, 1987.

5. Mullan F. Community-oriented primary care: an agenda for the '80s. N Engl J Med 1982;307:1076-8.

6. Tucker TC, Friedell GH. Using cancer registry data in primary care practice. Prim Care Cancer 1994;14: 33-6.

7. Williams RL, Flocke SA, Zyzanski SJ, Mettee TM, Martin KB. A practical tool for community-oriented primary care community diagnosis using a personal computer. Fam Med 1995;27:39-43.

8. Alpha 4, 3.1. Burlington, Mass: Alpha Software Corp, 1993.

9. MapInfo for Windows, version 2.1.1. Troy, NY: MapInfo Corp, 1992.

10. Snow J. On the mode of communication of cholera. 2nd ed. London: Churchill, 1855. Reproduced in: Snow on cholera. New York: Hafner Press, 1965.

11. Pickles WN. Epidemiology in country practice. Baltimore: Williams \& Wilkins, 1939.

12. Mullan F, Nutting PA. Primary care epidemiology: new uses of old tools. Fam Med 1986;18:221-5. 